\title{
Contraception et planning familial dans la Grèce antique
}

\author{
Georges ANDROUTSOS
}

Histoire de la Médecine, Faculté de Médecine, Université D’loannina, Grèce

\section{RÉSUMÉ}

Les Grecs anciens ont utilisé diverses méthodes de contraception, telles que le coitus interruptus, le coitus reservatus, le coitus obstructus, le coït anal, l'application des pommades sur le pénis, les médicaments, la magie, etc. Dans le cadre du planning familial, pour avoir des familles peu nombreuses, ils n'ont pas hésité de recourir à l'interruption de la grossesse (avortement), à l'infanticide ou à l'exposition.

Mots clés : Contraception, planning familial, interruption de grossesse, infanticide, exposition

\section{INTRODUCTION}

Cette histoire de la contraception en Grèce antique étudie tout particulièrement l'expérience des efforts des Grecs anciens pour contrôler le nombre de leurs enfants. Cette étude nous enseigne que l'idée de limiter la descendance a toujours été présente. Beaucoup de pratiques contraceptives restent en vigeur depuis plus de deux mille ans.

\section{METHODES CONTRACEPTIVES}

Lorsqu'on étudie les différents procédés de « contraception » qui ont pu être imaginés et décrits tout au long de l'histoire de l'humanité il importe de toujours garder présent à l'esprit les notions de physiologie de la reproduction que possédaient les peuples à l'époque concernée. Ainsi était considéré comme "contraceptif » par les médecins de l'Antiquité tout procédé applicable avant la « conception du fœetus », c'est-à-dire pratiquement avant le troisième mois de grossesse.
Pour bien comprendre les théories des Grecs sur le contrôle de la fécondité, il faut se rappeler qu'ils croyaient à la possibilité d'influencer la procréation. Les Grecs considéraient ces problèmes comme les deux faces d'une même monnaie. Quand on abordait le problème de la stérilité, on mettait en garde les femmes contre les risques que faisait courir à leur santé l'absence de rapports sexuels. Mais quand les hommes choisissaient de limiter ou d'éviter la paternité, on les rassurait en leur affirmant que leur abstinence était à la fois prudente et saine. «Le coït, assurait Démocrite, est une légère attaque d'apoplexie. Car l'homme jaillit de l'homme et n'est pas loin d'être déchiré par une sorte de choc » [10]. La semence, selon les pythagoriciens, était une « goutte de cervelle qui contient en soi de la vapeur chaude » [9] et tout plaisir sexuel était donc nuisible. Platon, Aristote et le Corpus hippocraticum [14] insistaient aussi sur la nécessité de pratiquer la modération sexuelle.

Ceux qui trouvaient l'abstinence difficile à pratiquer, mais qui tenaient néanmoins à restreindre leur fécondité, devaient avoir recours à d'autres méthodes. Le coït interrompu constitue certainement le moyen contraceptif le plus ancien et le plus repandu à travers le monde. Plusieurs allusion à cette pratique figurent dans la Bible, la plus connue étant bien entendu l'histoire d'Onan. Le coït interrompu était, et demeure, parmi les formes les plus simples de contraception, mais il n'y est fait que rarement allusion dans la Grèce ancienne. Platon l'évoquait peut-être quand il déclarait «qu'on ne jette pas la semence parmi les rocs et les cailloux où elle ne prendra jamais racine ». C'est

\section{Correspondance :}

G. Androutsos, 1, rue Ipeirou, 10433, Athènes, Grèce. Fax : 01-8235710.e-mail : paris48@otenet.gr. 
peut-être aussi ce que voulait signifier Hérodote quand il mentionnait le cas de Pisistrate, le tyran d'Athènes : « Comme il avait des fils adolescents, et que les Alcméonides passaient pour être maudits, il ne voulait pas qu'il lui naquît des enfants de sa nouvelle épouse et n'avait avec elle qu'un commerce contre nature »[20]. C'est dans la poésie qu'on a trouvé les allusions les plus nombreuses au retrait. Archiloque de Paros (poète du VIIème siècle av. J.-C.), semblait bien faire allusion au coït interrompu, quand il décrit sa relation avec la sœur de sa fiancée : « J'emmenai alors la fille parmi les fleurs et la couchai sur le sol. Alors, sur le champ elle me dévoila sa jeune chair tout en caressant son corps charmant, je laissai aller ma force, effleurant tout juste son duvet fauve » [16]. Si le coït interrompu était, dans le monde antique, comme il devait l'être dans l'Europe du XIXème siècle, la principale forme de contraception, il est possible qu'on n'y ait fait que rarement allusion parce que ni le besoin ni l'occasion ne se faisaient sentir de le décrire ou simplement de l'évoquer. Il se peut aussi que le coït interrompu - parce qu'il exigeait de sacrifier un plaisir auquel peu d'hommes étaient prêts à renoncer - n'était pas couramment pratiqué. L'extraordinaire valeur accordée à la semence mâle par Aristote justifiait assurément l'hostilité des hommes à recourir à de telles méthodes. Mais, étant donné l'étendue des connaissances des Grecs érudits sur le processus de procréation il est difficile de croire qu'ils ignoraient l'efficacité de cette méthode.

Contrairement au coït interrompu, il n'y pas d'émission de sperme lors du coïtus reservatus, le pénis étant retiré avant l'orgasme.

Coïtus obstructus est un procédé dangereux, consistant à comprimer l'urèthre au moment de l'orgasme de façon à provoquer une éjaculation rétrograde.

Par rapport au condom, il est difficile de fixer avec certitude la date de son apparition dans l'histoire de la régulation des naissances. La première description écrite du condom est celle de Gabriel Fallope en 1564. Selon la légende, le sperme de Minos, roi de la Crète, contenait des graines de serpents et de scorpions, ce qui l'empêchait de procréer ; et son union avec Pasiphaé demeurait donc stérile. Prokris, fille d'Erechteus, s'étant querellée avec son mari Cephalus se réfugia en Crète. Elle eut l'idée de façonner en gaine une vessie de chèvre ; cet objet, selon Antonin Liberalis, auteur romain d'un ouvrage intitulé Les Métamorphoses, fut introduit dans le vagin d'une femme. Minos put ainsi s'y débarrasser du contenu encombrant de son sperme, puis aller avec Pasiphaé, et procréer. Il eut de cette façon huit enfants. Selon Liberalis, la vessie de chèvre était utilisée par la femme et constituait donc un précurseur du diaphragme. Selon une autre hypothèse Liberalis s'est trompé et c'est en réalité Minos qui a utilisé l'objet. Celui-ci serait alors le précurseur du condom. Par ailleurs Les Métamorphoses ont été écrites à l'époque romaine et les Romains utilisaient effectivement déjà des préservatifs masculins [19].

Hippocrate recommandait, si la grossesse n'était pas désirable, de faire l'amour pendant les jours inféconds. Une autre méthode était le coït pendant la menstruation. Il faut signaler ici que les Grecs ne considéraient pas que l'acte sexuel pendant la menstruation était quelque chose d'impur dont il devaient se purifier après [21].

La pénétration non vaginale ne donnerait évidemment pas lieu à la procréation. L'enthousiasme des Grecs pour l'homosexualité a fort bien pu populariser les rapports anaux dans les relations hétérosexuelles. K.-J. Dover a remarqué que, quand l'art grec dépeignait des scènes hétérosexuelles, il s'agissait souvent de sodomies. Il se demande également si les hétaïres (ou courtisanes) n'employaient pas les rapports anaux comme méthode de contraception [7]. Par ailleurs, ni le Coran ni le Talmud n'interdisent les rapports anaux avec l'épouse [12].

$\mathrm{Si}$ le retrait est classé comme forme « masculine » de contraception, on peut considérer comme des formes « féminines » l'emploi de pessaires obstructeurs, de tampons et de potions. Les sources, dans ce domaine sont plus nombreuses et elles prouvent que la limitation des naissances était principalement l'affaire des femmes [8]. Malheureusement, les textes écrits par des hommes ne fournissent nécessairement qu'une image un peu vague des actions et des mobiles des femmes. Puisque l'allaitement arrête l'ovulation, la façon la plus « naturelle » dont les femmes auraient pu se protéger d'une nouvelle grossesse suivante aurait été de prolonger la période durant laquelle elle nourrissait au sein un premier enfant. Il semble toutefois que les femmes grecques de la haute société confiaient leurs enfants à des nourrices, ce qui signifiait qu'elles devaient chercher d'autres formes de protection [11].

On trouve ainsi des allusions à des mouvements corporels précis, pendant et juste après le coït, susceptibles d'inhiber ou au contraire de favoriser la fécondation. Dans De la Génération, un passage évocateur précise : « Après le coït, si la femme ne doit pas concevoir, elle fait d'habitude tomber au dehors, quand elle veut, la semence provenue des deux individus » [13].

Les Grecs croyaient qu'un certain nombre de potions à base d'herbes avaient un effet contraceptif. Ils désignaient le contraceptif par le mot atokion. On ne savait néanmoins pas toujours clairement si une décoction avait pour objet de prévenir la conception ou d'y mettre un terme. Le traité $D e$ materia medica [6] de Dioscoride (Ier siècle ap. J.-C.), complétant les informations sur les tisanes fournies par Théophraste (IVème siècle av. J.-C.), énumérait de nombreuses recettes de breuvages censés contenir des éléments stérilisants. Une espèce de choux, macéré dans du lait de chèvre, devait exciter l'élan sexuel et le diminuer si on le mangeait sec. Les baies de genièvre placées sur le pénis ou 
dans la vulve produisant, affirmait-on, une stérilité temporaire.

La première mention d'application d'une substance sur le pénis remonte au Ier siècle ap. J.-C. : Pline parle d'appliquer de l'huile ou de la résine de cèdre, transposant à l'homme une recette préconisée auparavant par Aristote pour la femme. Oribase recommande « d'oindre la partie virile de l'homme avec du jus d'hedysome » avant le rapport sexuel. Aëtius recommande d'enduire le pénis avec des astringents, alun, grenade, ou noix de galle, triturés dans du vinaigre, ou encore des lavages avec de la saumure. La Misy (sulfate de cuivre), était recommandé dans les ouvrages hippocratiques. Des potions venaient renforcer les diverses méthodes de contraception mécanique. Dioscoride conseillait d'oindre les organes génitaux avec de la gomme de cèdre et d'appliquer de l'alun sur l'utérus. Dioscoride mentionnait également un suppositoire à la menthe poivrée et au miel avant le coït et un pessaire poivré à utiliser après le coït. Selon la théorie hippocratique, ces suppositoires étaient efficaces, non pas parce qu'ils constituaient une barrière mais parce qu'ils desséchaient l'utérus [15].

Les femmes grecques ont pu tenter d'employer la méthode des cycles. On croyait que la conception avait des chances de se produire soit juste avant, soit juste après la menstruation. Dans le livre De la Génération on trouve que les courtisanes savaient quand elles avaient conçu. [17] A part des diverses substances (p.e. sulfate de fer, plomp argentifère, nitrate de potassium et d'autres poisons avec des qualités spermicides et/ou contraceptives, véritables ou imaginaires) et si tout le reste échouait, on pouvait avoir recours à la magie sous forme d'amulettes et de talismans protecteurs. Les partisans de la magie affirmaient que des décoctions à base de rognons de mulets et d'urine d'eunuques rendaient aussi impuissant [6]. Selon Dioscoride, lorsqu'une femme enceinte marchait sur une racine de cyclamin, elle aurait une fausse-couche. Toujours selon le même auteur, celui qui portait sur lui une racine d'asperge, deviendrait provisoirement stérile ! Dans l'Histoire Naturelle de Pline est écrit que lorsqu'une femme enceinte mangeait un œuf de corbeau, elle aussi aurait, une fausse-couche !

\section{PLANNING FAMILIAL}

Dans le monde antique, les familles étaient relativement peu nombreuses. Cela tenait à une variété de causes. La plupart des familles ne désiraient pas un grand nombre d'enfants pour que la fortune de la famille ne fût pas partagée entre de nombreux héritiers. De plus, les filles étaient, évidemment, moins désirables que les garçons car, d'une part elles ne travaillaient pas et ne produisaient pas et de l'autre part elles devaient prendre une dot en se mariant, ce qui réduisait la propriété familiale. Selon Platon [20] le nombre idéal des enfants d'une famille était deux : un garçon et une fille. L'espérance de vie était brève (45 ans pour les hommes et 36,2 pour les femmes). Les taux de natalité et de mortalité oscillaient chaque année entre 35 et $50 \%$. On ne pouvait donc espérer qu'un accroissement modeste de la population [2]. Bien des hommes choisissaient de ne pas se marier. Ceux qui le faisaient avaient peu d'enfants. Compte tenu de l'âge précoce auquel les femmes se mariaient cinq à six naissances étaient possibles. Mais on a estimé qu'en moyenne, il ne naissait que quatre enfants par famille, et que deux ou trois seulement survivaient. Les raisons pour expliquer ces chiffres faibles sont les suivantes. L'une est la tolérance générale, voire l'approbation à l'égard de l'homosexualité masculine. En Crète, selon Aristote, l'homosexualité bénéficiait d'un soutien officiel : on y voyait un moyen de contrôler le développement de la population [3]. Les hétérosexuels résolus avaient le choix entre plusieurs options. Ils pouvaient notamment recourir aux services des courtisanes. Même si des enfants étaient conçus, la malnutrition et les maladies qui frappaient les mères aboutissaient sans doute à un nombre important de morts parmi les fœtus. La petite taille des familles était donc la conséquence tout à la fois d'un taux de mortalité élevé et des pratiques de restriction de la fécondité. Un grand nombre d'hommes et de femmes pensaient que la fécondité devait et pouvait être contrôlée. Mais, étant donné la nature de la famille antique, les hommes et les femmes auraient fort bien pu avoir des raisons différentes et employer d'autres méthodes pour atteindre ce but. On trouve une critique de la famille nombreuse dans la condamnation des excès sexuels prononcée par Platon et par les stoïciens. Platon et Aristote comptent parmi ceux qui préconisèrent le contrôle du mariage par l'État et l'eugénisme à l'encontre des enfants inaptes. Dans La République, Platon évoque la nécessité de réguler la fécondité, de sorte que la progéniture n'excède pas les moyens de la communauté. Dans Les Lois, il envisage d'assurer la stabilité du nombre de détenteurs de terres en n'ayant qu'un héritier par famille [20]. A son tour, Aristote suggère que l'on réglemente l'âge du mariage et qu'on ait recours à l'avortement [4].

Les Grecs considéraient que leur système familial ne fonctionnait que s'il y avait des héritiers légitimes, mais pas en trop grand nombre. La méthode la plus simple pour parvenir à ce but aurait été de reculer l'âge du mariage des femmes et de limiter ainsi le nombre d'années où elles pouvaient enfanter. Mais, parce qu'on voulait assurer la domination masculine, on mariait les femmes à un âge précoce et on pouvait donc s'attendre à ce qu'elles enfantent de nombreuses fois.

\section{AVORTEMENT ET INFANTICIDE}

Pour de nombreuses sociétés, la signification du mot avortement n'était pas la même que celle que nous lui attribuons actuellement. Ce fait est lié au degré de connaissances dans le domaine de la reproduction.

Pour les Grecs, la vie naissante n'avait que des droits limités. L'opinion générale voulait qu'un fotus ne fût jamais 
plus qu'un être humain en puissance. Les stoïciens partageaient cette opinion en le considérant jusqu'à sa naissance comme une partie de la mère. L'avortement présentait donc un problème moral moins délicat pour les Anciens que pour nos contemporains, dans la mesure où ils supposaient que la vie n'apparaissait qu'avec la venue au monde [5]. Selon Aristote, le fotus prend qualité d'être humain vers le $40 \mathrm{e}$ jour après la conception pour un garçon, vers le $90^{\mathrm{e}}$ pour une fille. Pour Hippocrate, les premiers jours qui suivent la conception correspondent simplement à la retention par la femme de la semence de l'homme. Faire sortir cette semence ne constitue pas un avortement mais une effluxion. Le terme d'embryon ne convient qu'au fotus animé. Hippocrate désigne ses préparations médicales en fonction de leurs effets supposés : « pour détruire et chasser le fotus qui ne bouge pas », ou encore « pour amener les règles et tirer le fœus à demi formé ». Ainsi peut-on expliquer qu'il n'y ait pas de contradiction entre les conseils donnés par Hippocrate à une courtisane pour avorter (il s'agissait d'une semence de six jours) et son Serment dans lequel il est écrit : « Je ne donnerai à personne, même sur sa demande, un médicament mortel, ni ne donnerai de conseils en ce sens ; de la même façon, je ne donnerai à aucune femme de pessaire abortif. " Hippocrate insiste d'ailleurs, dans son ouvrage Des maladies des femmes sur les dangers d'un avortement. C'est à Soranos que revient le mérite d'avoir clarifier nettement les notions de contraception et d'avortement dans son traité Gynaecia : « La contraception, atokion, diffère de l'avortement, phtorion, en ceci que la première désigne un remède qui prévient la conception, alors que le second, au contraire, désigne un remède qui tue le fœtus » Ailleurs, Soranos précise : «...Un efflux est une expulsion de semence un, deux ou trois jours après les rapports ; un avortement est la destruction de l'embryon au deuxième ou troixième mois ; un accouchement prématuré est sa mise au monde tout près du terme, mais avant le moment opportun. »

Certains pensent que l'expulsion, ekbolion, est synonyme d'avortement ; d'autres cependant disent que contrairement à l'avortement, l'expulsion ne désigne pas une médication mais au contraire une agitation violente du corps, comme sauter par exemple. Ainsi Hippocrate dans son livre De la nature de l'enfant a rejeté les abortifs et préconisé une méthode pour déclencher l'avortement en sautant de façon à ce que les fesses soient touchées avec les pieds. Les opinions concernant l'utilisation des abortifs divergent cependant. Beaucoup les rejettent, se référant au Serment hippocratique : «Je ne prescrirai jamais un abortif » et déclarant en outre que le but de l'art médical est de prescrire et de sauver les œuvres de la nature. D'autres autorisent l'utilisation d'abortifs dans des cas exceptionnels.

\section{Interruption de grossesse}

La grossesse imprévue était autant indésirable pour les femmes mariées que pour les courtisanes et les prostituées
[21]. Les relations extra-conjugales du mari suffisaient à satisfaire, voire saturer son instinct sexuel, de sorte qu'il couchât rarement avec sa femme. Cependant, en cas de grossesse indésirable, la femme devait choisir entre la fausse-couche volontaire, en pratiquant des sortilèges, et l'avortement. Et même si c'était à elle de décider en cas de grossesse indésirable, en ce qui concerne l'avortement, le consentement de son mari - ou du maître de l'esclave - était indispensable.

L'avortement a été pratiqué par toutes les sociétés anciennes. L'avortement, par absorption orale de substances diverses, par introduction vaginale de tampons ou pessaires variés, ou par manœuvres intra-utérines, se retrouve dans toutes les sociétés étudiées, qu'elles soient primitives ou «civilisées », et quelle que soit l'époque. Les allusions à l'avortement sont bien plus fréquentes que les références à la contraception. Il y avait à cela de bonnes raisons. La contraception - notamment le coït interrompu, la pratique d'une sorte de méthode des cycles ou les rapports anaux - était un acte intime qui, le plus souvent, n'était ni révélé ni enseigné. L'avortement, en revanche, exigeait souvent une assistance ou des conseils.

Les Grecs disposaient de nombreuses techniques d'avortement, y compris l'usage de perforations, de pessaires, de potions orales, de suppositoires, de fumigations et de cataplasmes. Les sauts, les coïts répétés, les fièvres, les vomissements et les saignements étaient décrits comme facteurs des fausses couches [12]. Les médecins savaient que les fausses couches pouvaient être provoquées mais ils ne se livraient à ces pratiques qu'avec beaucoup de prudence. L'avortement a été décrit comme une pratique à laquelle les femmes avaient parfois secrètement recours mais on ne trouve aucune trace de loi contre l'avortement dans les textes des premiers législateurs comme Lycurgue de Sparte ou Solon d'Athènes. Platon réclamait, dans le cadre d'une politique d'État, l'avortement pour toutes les femmes ayant conçu après l'âge de quarante ans. Si les Grecs toléraient le déclenchement de fausses couches, on peut se demander pourquoi le Serment hippocratique contenait sa fameuse injonction interdisant aux médecins de pratiquer l'avortement. Pour certains, il s'agit en fait d'une addition pythagoricienne tardive au Corpus. Le culte pythagoricien, hostile à la chirurgie, allait à l'encontre d'un grand nombre de théories et de pratiques médicales grecques. Selon d'autres [1], l'injonction contre l'avortement pourrait signifier seulement que les médecins devaient en général laisser aussi bien les naissances que les avortements sous la juridiction des sages femmes. On trouve cependant dans le Corpus une technique d'avortement recommandée par un médecin [14].

\section{Infanticide, exposition (abandon)}

Hippocrate, posait la question «quels enfants il convient d'élever ». Soranos définissant la puériculture comme l'art de décider « quels sont les nouveau-nés qui méritent qu'on 
les élève ». Platon, dans La République, écrit : «Il faut élever les enfants des premiers, non ceux des seconds si l'on veut maintenir au troupeau son excellence. » A partir du moment où un enfant était né et était indésirable, soit parce qu'il était illégitime soit parce que la famille en avait déjà trop et un de plus serait un charge (le père attendait quelques jours avant d'annoncer publiquement s'il fallait ou non conserver son enfant), il y avait deux façons pour se débarrasser de lui : le tuer ou l'exposer.

Solon (VIème siècle av. J.-C.) fit publier une loi reconnaissant le droit à l'infanticide [19]. Certes, bien que l'infanticide comptasse parmi les actes criminels, il était tout de même pratiqué. A Sparte, c'était l'État qui s'en chargeait, mais pour des raisons différentes : les nouveaux-nés étaient présentés aux Anciens de la tribu qui les examinaient ; ceux qui étaient mal venus et difformes étaient emmenés au mont Taygète (Plutarque, Lycurgue, 16) où ils étaient précipités dans un gouffre appelé "Apothètes ». Encore une preuve de l'eugénisme spartiate !

Une méthode beaucoup plus répandue pour se débarrasser de l'enfant indésirable était l'exposition qui, la plupart du temps, entraînait la mort faute de nourriture et de soins. Toutefois, l'exposition devait avoir lieu les premiers jours après la naissance de l'enfant et surtout avant le dixième jour où, à Athènes, l'enfant recevait son nom. En effet, c'était à partir de ce moment là que, selon les Grecs, l'enfant commençait à exister dans la société. Les peu nombreux qui survivaient à l'exposition, étaient automatiquement considérés commes des esclaves et les filles finissaient comme protituées. Cenpendant, certains enfants avaient de la chance, étant sauvés et élevés par ceux qui les avaient trouvés.

\section{CONCLUSION}

Les Grecs anciens ont eu recours à la contraception et au planning familial par diverses méthodes. Certaines d'entre elles sont encore en usage, d'autres, par contre, nous paraissent aujourd'hui primitives ou même atroces et ont été abandonnées au cours des siècles.

\section{RÉFÉRENCES}

1. ANDROUTSOS G.: L'histoire du préservatif. Andrologie, 4, 1994, 492-504.

2. ANGEL L. : The basis of Paleodemography. Am. J. Phys. Anthropol., 30, 1969, 427-438.

3. ARISTOTE. : Politique. Texte établi et traduit par Jean Aubonnet, Les Belles Lettres, Paris, 1960, Livre 2, 10, 1272a.

4. ARISTOTE. : Politique. Texte établi et traduit par Jean Aubonnet, Les Belles Lettres, Paris, 1960, Livre 7, 16, 1335b.

5. DICKINSON SH : Abortion in Antiquity. Arethusa, 6, 1973, 159-160.

6. DIOSCORIDE : De materia medica, 1. 104.
7. DOVER K.-J. : Greek Homosexuality, 100-1.

8. FONTANILLE M-T: Avortement et contraception dans la médecine gréco-romaine, Searle, Paris, 1977, 34-8.

9. FOUCAULT M. : Histoire de la sexualité (II). L'Usage des plaisirs. Gallimard, Bibliothèque des Histoires, Paris, 1984, p. 146.

10. FREEMAN K. : Ancilla, fragment 32, p. 99.

11. GOLDEN M. : The effects of slavery on citizen households and children, historical reflections, 15, 1988, p. 456.

12. HIMES N.E. : Medical history of contraception, Gamut Press, New York, 1963, p17

13. HIPPOCRATE : De la Génération, 10, 1. p. 477.

14. HIPPOCRATE : De la Nature de l'enfant, 13, p. 491.

15. HIPPOCRATE : De la Nature de la femme, Littré, p. 415.

16. LEFKOWITZ M. FANTEDS M. : Women in Greece and Rome, Samual Stevens, Toronto, 1977, p. 74

17. LOYD G. E. R. : Science, Folklore and Ideology : Studies in the Life Sciences in Ancient Greece, Cambridge University Press, Cambridge, 1983, p. 78.

18. MCLAREN A. : Histoire de la contraception, Noêsis, Paris, 3366.

19. NETTER A., ROZENBAUM H.: Histoire illustée de la contraception, de l'Antiquité à nos jours, Dacosta, Paris, 1985, 318320.

20. PLATON. : Les Lois, Livre $7,838^{\mathrm{e}}$, dans Platon, Euvres Complètes, texte établi et traduit par A. Diès, Paris, Les Belles Lettres, t. XII, 1956 ; Hérodote. Histoires, Livre 1, 61.

21. VRISSIMTZIS N.: Amour, sexe et mariage en Grèce antique. Vrissimtzis, Athènes, 1996, 47-49.

\section{ABSTRACT}

\section{Contraception and family planning in ancient Greece}

\section{Georges ANDROUTSOS}

The ancient Greeks used various methods of contraception, such as coitus interruptus, coitus reservatus, coitus obstructus, anal coitus, the application of ointments onto the penis, medicines, sorcery, etc. In the context of family planning, to avoid large families, they also resorted to termination of pregnancy (abortion), infanticide and exposure.

Key words: Contraception, family planning, termination of pregnancy, infanticide, exposure. 